\title{
La historia, las ciencias sociales y la Universidad de Sonora como objeto de estudio
}

HISTORY, SOCIAL SCIENCES AND THE UNIVERSITY OF SONORA AS AN OBJECT OF STUDY

\author{
Armando Moreno Soto ${ }^{1}$
}

\section{Resumen}

En este trabajo de investigación, se presenta el estado del arte del cambio y continuidad institucional en la historia de la Universidad de Sonora: 19381982, mediante un balance de los libros, capítulos, artículos de revistas, ponencias e incluso en algún momento artículo de periódico, que tiene por objeto de estudio la Universidad de Sonora ya sea desde la historia o de otra ciencia social.

Palabras clave: Universidad de Sonora, continuidad institucional, historia.

Recibido: 14 de junio del 2017.

Aceptado: 18 de diciembre del 2018.

\begin{abstract}
In this paper, its present the state of the art of change and institutional continuity in the history of the University of Sonora: 1938-1982, through a balance of books, chapters, articles of magazines, papers and even at some point Newspaper article, which is intended to study the University of Sonora either from history or other social science.
\end{abstract}

Keywords: University of Sonora, institutional continuity, history.

\footnotetext{
${ }^{1}$ Armando Moreno Soto. Doctor en Ciencias Sociales. Presidente de la Academia de Historia Económica del Departamento de Economía. Universidad de Sonora. E-mail: amorenosguaymas.uson.mx
} 


\section{Introducción}

La escritura de la historia de la (Unison) -más allá de las actas y documentos oficiales, de la información en los periódicos locales o nacionales, de lo publicado en revistas o el periódico universitario, de los programas de desarrollo, de los discursos de los funcionarios universitarios o gubernamentales, de los edificios construidos, de los murales pintados y de todo aquello que, como fuente primaria, una institución va dejando como huella de su paso a través del tiempo-, necesita de una actitud reflexiva que implica recopilación de información, análisis e incluso síntesis, de manera coherente, racional, con el fin de reproducir o, en un mejor caso, explicar el hecho o el proceso, lo cual es todavía una empresa más joven que la propia universidad.

Hasta 1967, cuando la universidad cumplió 25 años de haber abierto sus puertas y 29 de haber sido creada formalmente, solo un documento cumplía con el carácter de obra histórica: la Monografía histórica de la Universidad de Sonora del maestro y tercer rector, Manuel Quiroz Martínez (1949), que fue presentada como ponencia con el título Los primeros tiempos de la Universidad de Sonora en la novena sesión plenaria del Congreso Mexicano de Historia, realizado en Hermosillo.

De 1967 en adelante y, sobre todo, a partir de la década de los ochenta, los trabajos que tienen por objeto de estudio la universidad y a los universitarios, sean estudios de historia con su narrativa como actor principal o la historia como complemento al análisis de un fenómeno particular, comienza a ser una actividad más cotidiana, sistemática.

\section{Trabajos de historia general de la Universidad de Sonora}

Como historia general de la Unison existen dos trabajos que son, por su dimensión, con mucho, los más notables: el primero, Historia de la Universidad de Sonora de Miguel Castellanos Moreno (1981, c1991; 1992; 2007; 2006), de profesión historiador y Maestro de Tiempo
Completo de la propia universidad. El segundo, Historia General de la Universidad de Sonora de Carlos Moncada Ochoa, abogado, periodista, maestro por tiempos en la universidad y escritor. En el caso de Miguel Castellanos se trata de cuatro tomos editados en el transcurso de veinticinco años (1981 el primero y 2006 el cuarto, el tercer un año después en Jalisco) en su parte sustantiva con peculio personal, apoyo familiar y del STAUS; se encuentra inconclusa, pues el último trabajo llega hasta los ochenta. Carlos Moncada Ochoa (2005; 2006; 2007; 2009; c2009), en cambio, acometió la tarea a solicitud expresa del entonces rector, Pedro Ortega; la institución editó el trabajo en cinco tomos, en algo así como la historia oficial, de los cuales el segundo es una recapitulación de leyes que debería estar en un apéndice y no cómo parte constitutiva de la obra.

Otros trabajos menores con la pretensión de hacer historia general de la Unison son: el capítulo que le dedican (Universidad de Sonora) Jaime Castrejón Diez y Marisol Pérez Lizaur (1976) en su historia de las universidades estatales. Un año antes, en Hermosillo, José Abraham Mendívil, creador del periódico El Imparcial, fundamental como líder sindical y miembro del partido oficial, central en la expulsión de los vasconcelistas, publicó años después La Universidad de Sonora (desde sus orígenes [1975]), donde, además de algunos documentos básicos, sobre todo artículos de periódicos y cartas, se pronuncia de izquierda, según su decir, cuando el enfrentamiento con los vasconcelistas; en cambio, se horroriza ante los movimientos y el activismo estudiantil de izquierda de finales de los sesenta y principios de los setenta, con una posición de derecha. Otro documento es la ponencia de Rodolfo Díaz Castañeda (1987), Esbozo histórico de la Universidad de Sonora, basada en las leyes orgánicas y otros datos fundamentales. También el apartado "Las instituciones educativas” de Eduardo Ibarra y Ernesto Camou Healy, en el V tomo (Historia contemporánea de Sonora, 1929-1984), de la Historia General de Sonora. 
De un servidor están en este paquete, "Universidad de Sonora: cuarenta y cinco años de vida” (1988), un artículo de Revista de la Universidad de Sonora, y el folleto Historia del desarrollo de la Universidad de Sonora (1991), donde se ha venido experimentando con la periodización y una versión particular.

La mayoría de estos trabajos tienen en común ser historia política, es decir, tratan de la relación de la universidad y los universitarios con el poder: con el grupo gobernante y el Estado nacional representado en lo inmediato por el Gobierno de Sonora y la clase dominante, expresada en los empresarios locales, incluyendo agricultores y ganaderos; además de los eventos políticos internos, gobierno, personajes (especialmente rectores), acontecimientos que van al unísono con la relación con el exterior. Naturalmente, esta historia es muy valiosa, incluso la que tiene claro propósito propagandístico o apologético.

\section{Historias de unidades académicas}

En los últimos años comenzaron a ser editadas. Luis Enrique García (1992) publicó Siete notas para las Bellas Artes, una imprescindible historia de las Academias. Anteriormente (1984), del mismo autor, se había publicado Memoria teatral de la UniSon, 19541984. Posteriormente, el ingeniero y maestro universitario, Manuel Sortillón Valenzuela, dio a conocer Memorias de la Universidad de Sonora y de su Escuela de Ingeniería (1998). Otro libro cuyo objeto de estudio es la historia de una unidad académica es de Alberto Macías Enríquez, Historia de la Facultad de Derecho de la Universidad de Sonora del 2006. También, Historia de la escuela de Medicina de la Universidad de Sonora del 2000 al 2010. Raíces médicas e históricas del Dr. Alfredo Padilla Barra (2013). Como libro conmemorativo, Ingeniería química: 50 años. Memoria 1962-2012, realizada y publicada (2012) por la Unison, bastantes fotos y texto indispensable. En ese mismo tenor, 50 años de Ingeniería Industrial en Sonora, de Jorge Taddei Bringas y Ricardo Rodríguez Carvajal (2013), editado también por la Unison. A tres años de constituida la Editorial de la unidad centro (Hermosillo), por la entonces Vicerectora, Dra. Guadalupe García de León, la Mirada del Búho comienza a rendir frutos. El primero, Departamento de Lenguas Extranjeras: una visión de su desarrollo en la Universidad de Sonora, de septiembre del 2015 con la autoría de la maestra Sofía Dolores Cota Grijalva. En diciembre de ese mismo año, el investigador y maestro Ricardo Rodríguez Mijangos a través de la misma convocatoria dio a conocer, Memorias Alrededor de la Investigación Científica en Física en la Unison.

\section{Historia de momentos específicos de la historia de la Unison}

Sobre el origen de la universidad se encuentra el testimonio de un participante en el proceso vasconcelista, directamente fundador -incluso miembro del comité administrativo-, quien fuera muchos años después, en 1967, candidato del PAN a la gubernatura, Lic. Gilberto Suárez Arvizu, con un artículo titulado "Universidad de Sonora: antecedentes históricos", publicado en la Revista de la Universidad de Sonora en abril de 1983. Por otra parte, en el periódico Información, de 13 de octubre de 1982 al 18 de marzo del año siguiente, el doctor en derecho, Domingo Gutiérrez Mendívil, publicó un artículo con mucha información sobre el origen y datos complementarios "La Universidad de Sonora en sus Orígenes". Por último, mencionaremos el trabajo de Luis Felipe Larios Velarde, Enrique Gurrola Mac y Jesús Alejandro Salido Ontiveros (1985), Notas históricas sobre la creación de la Universidad de Sonora en 1938, realizado como trabajo escolar de una maestría en Administración.

Del llamado movimiento estudiantil y popular de 1967 existe mayor reflexión. Ese mismo año desde la Ciudad de México, el prolijo escritor nogalense, Óscar Monroy Rivera, publicó Sonora en torno al valor de mi pueblo, un trabajo muy pobre en cuanto a datos e interpretación; Luis Encinas Johnson (1969), a quien correspondió presidir el Ejecutivo estatal en aquel momento y exrector de la Unison, dio su versión de los acontecimientos en La alternativa de México (conflictos, causas y caminos), vale la pena mencionar que no cae en la descalificación comunista -muy común entonces-, 
el conflicto lo atribuye a errores en el procedimiento interno priista de selección del candidato; lo ubica dentro del conjunto de conflictos de aquella época, específicamente como enfrentamiento entre generaciones y como parte de la problemática general del país, sin dejar de ser siempre un hombre del sistema, del partido oficial. Por el contrario, un activo participante de aquellos avatares como estudiante, Ismael Mercado Andrews (1973), publicó en folleto su testimonio, El día que estalló la rabia, reeditado posteriormente por la Unison en 1997. El más importante de los historiadores políticos en Sonora en la segunda mitad del siglo XX, Carlos Moncada Ochoa, tiene dos obras que abordan en extenso el tema, Años de violencia en Sonora de 1976 y la ya referida Historia de la Universidad de Sonora.

Por otra parte, los días 26, 27, 28 y 29 de septiembre de 1983, aparecieron en el periódico Información el artículo de Armando Moreno Soto intitulado El movimiento estudiantil y popular de 1967 en Sonora; dos años después, el mismo autor y otros, publicaron el libro Los aguiluchos: movimiento estudiantil y popular de 1967 en Sonora -mal que se diga pero más mal que ocurra-, hasta hoy el trabajo más completo sobre el movimiento social con mayor importancia en la segunda mitad del siglo XX y con epicentro en la Unison, cuyos protagonistas fundamentales son los estudiantes universitarios. En la memoria del XXII Simposio de Historia y Antropología de febrero de 1997, dedicado precisamente a conmemorar el trigésimo aniversario de dicho acontecimiento, tres de sus protagonistas expusieron las ponencias: El movimiento de 1967 ¿mito o verdadero moviendo social?, del ingeniero Jesús A. Larios Gaxiola; ¿Rabia incontenida o candor desbordado?, del también ingeniero civil, José Luis Jardines Moreno; y Crónica de un domingo -del domingo de 14 de mayo, cuando la turba intentó sacar a los presos y sitió la comandancia de policía-, escrito por el matemático Jorge Ontiveros Almada, tres aguiluchos como se les llamaría a los líderes de aquel movimiento.

Curiosamente, la recuperación teórica del más complejo e ideologizado de los acontecimientos, el movimiento estudiantil-universitario de 1970-73, ha sido más lenta. Carlos Ferrá Martínez fue el primero con El movimiento Universitario Sonorense de 1973, un ensayo corto, escrito al calor del suceso por quien había sido el ideólogo y dirigente; en él debate con las fuerzas emergentes, al explicar la obvia crisis en que para entonces se sumía el movimiento (circuló mimeografiado). En esa misma condición circuló también el ensayo de Rubén Duarte Rodríguez, Universidad y luchas de clases, escrito a finales de los setenta. El que escribe a mitad de los ochenta expuso la ponencia El movimiento estudiantil-universitario de 1970-73 en uno de los simposios de Historia, pero quedó inédita al no entregar el escrito a la memoria, pero referida en obras como la de Joel Verdugo y en $\mathrm{Ya}$ no estoy para rosas de Rita Plancarte. Todos son ensayos cortos. El primer trabajo que aborda a profundidad el tema es la tesis de licenciatura en Historia de Gabriela Rosales González (1996), Historia de la Universidad de Sonora (reforma universitaria 1967-1973). Cuatro años después, Claudio Ayala Encinas (2000), quien fue uno de los últimos miembros de la Comisión Coordinadora de la FEUS, hizo público su testimonio-crónica que incorpora aspectos culturales, El fin de una ilusión. Rubén Duarte Rodríguez retomó y amplió el documento mencionado anteriormente y lo hizo libro, salió a la luz en 2003 con el título Días de fuego (el movimiento universitario sonorense de los años 70). En el mismo Simposio de Historia y Antropología, donde se conmemoró el trigésimo aniversario del movimiento estudiantil y popular de 1967, la maestra Manuela Guillén Lúgigo presentó la ponencia Los no protagonistas: un testimonio del movimiento estudiantil de 1973. El mejor trabajo sobre el movimiento estudiantil universitario de $1970-73$ es el de Joel Verdugo, El movimiento estudiantil en la Universidad de Sonora de 1970 a 1974 (un enfoque sociohistórico a partir del testimonio oral), presentado originalmente como tesis de maestría para El Colegio de Sonora (COLSON), fue publicado por el mismo Colegio en 2004. Del mismo Joel son también los ensayos presentados en el simposio XXV, La contracultura en Sonora: los azules y la nueva sensibilidad, para reencontrarnos con los hippies autóctonos enlazados al movimiento. 
Y en el simposio XXVI, Al fondo a la derecha: el Movimiento Mexicanista de Integración Cristiana (los micos) para ver la contraparte al movimiento encabezado por los activistas.

El primer texto publicado del movimiento estudiantiluniversitario de 1991-92 fue una Cronología de la reforma a la ley orgánica de la Universidad de Sonora, escrita por Ana Teresa Reyes. Otro académico de la propia universidad, José Luis Ramírez, fue el siguiente, al publicar mientras estudiaba una maestría en la Universidad de Arizona un documento intitulado The change of the university of Sonora law: a case análisis. Nolvia Cortez Román (1997) presentó una ponencia en el XXII Simposio de Historia y Antropología, Del desierto al Zócalo... Crónica del movimiento estudiantil de la Universidad de Sonora, 1991-1992.

\section{Biografías}

Las biografías son historias de personajes y, por tanto, un género dentro de la ciencia que da razón del pasado humano, no podemos dejar de mencionar el libro Nuestros rectores, edición conmemorativa del sexagésimo aniversario de la universidad -con investigación y redacción de Guadalupe Beatriz Aldaco (2002), cuyo crédito, malamente, apenas fue esbozado-; en dicho texto por primera vez se tiene concentrada en semblanzas bien logradas información fundamental desde el primer rector hasta Pedro Ortega, con la debilidad de la crítica mellada por el interés institucional.

\section{Memorias gráficas}

Existe, además, una serie de memorias gráficas donde no solo las fotografías hablan: Memoria Gráfica de la Universidad de Sonora (1992); Memoria gráfica del deporte universitario (1996); y Memoria gráfica del teatro universitario: 1854-2004 (2006). El motor de las tres memorias, en cuanto a los textos se refiere, ha sido Luis Enrique García, y Guillermo "Memo” Moreno en la fotografía (en el primer trabajo los textos son en coautoría). También, se encuentra impreso Universidad: espacio y arquitectura (memoria gráfica de la arquitectura de la Universidad de Sonora), en este caso con textos del arquitecto Jesús Félix Uribe García y con fotografías de Joel Montoya.

Hasta aquí, los escritos mencionados son históricos, narran un acontecimiento, un proceso, la historia de la Unison, de una unidad académica, de un conflicto, algunas son tan elementales como cronologías o monografías, otras en un nivel superior son resultado de una ardua interpretación; las hay desde ponencias hasta libros de amplia circulación. Lo importante a rescatar es la existencia de un acervo bibliográfico para nada desdeñable, que se fue construyendo, sobre todo, a partir del último tercio del siglo $\mathrm{XX}$, especialmente a partir de la década de los ochenta, escrito mayoritariamente por académicos de la misma institución.

\section{Otras ciencias sociales intervienen}

A dicho acervo se le deben sumar investigaciones sobre problemáticas ligadas a la universidad y los universitarios, analizadas a través de una teoría de las ciencias sociales diferente a la historia y que, por referirse al fenómeno en un orden de tiempo, aportan al conocimiento histórico. En ese tenor se encuentra De la concentración a la diversificación institucional. La educación superior en Sonora (2007), coordinado por J. Raúl Rodríguez Jiménez y Laura E. Urquidi Treviño, donde se constata desde la sociología cómo a nivel estatal, aunque con retardo, en los ochenta se reprodujo lo ocurrido en el mundo y la nación a partir de los sesenta y setenta: la masificación y diversificación de la educación superior (Raúl Rodríguez). Se hace una propuesta de clasificación del sistema de educación superior en Sonora (Laura Urquidi), se constata la expansión de la educación superior privada (Raúl Rodríguez y Juan Pablo Villalobos), hay un acercamiento a los centros de investigación en Sonora (Laura Urquidi y Andrea Sotelo) y a la profesionalización del trabajo académico en la Unison (Etty Haydeé Estévez). Del mismo Raúl Rodríguez J. es posible leer en la Revista Estudios Sociales (1997), un artículo que perfila la masificación y diversificación de la educación superior en Sonora: “Origen y desarrollo de la educación superior en Sonora”. Otro trabajo con orientación sociológica y de análisis comparativo es 
Estado, políticas, universidades en un período de transición (2000), de Adrián Acosta Silva, donde se analizan las reformas del periodo neoliberal en la Universidad de Guadalajara (UDG), la Universidad Autónoma de Puebla (UAP) y la Unison, a la que califica de "ajuste radical y diferenciación funcional”. Muy similar en la orientación -incluyendo el problema de la gobernabilidad, pero con un solo caso-, se encuentra un libro que fue premio ANUIES titulado Poder, gobernabilidad y cambio institucional en la Universidad de Sonora (1991-2001), cuyo resultado final es la justificación de la imposición de la Ley 4 orgánica y de las condiciones que hoy prevalecen. Otro tipo de casos relacionados lo podemos ver en el libro de Etty Estévez, Lo Doctorado no quita lo tarado, con el subtítulo, Pensamiento de académicos y cultura institucional en la Universidad de Sonora: significados de una política pública para mejorar la educación superior, donde con base en entrevistas hace una evaluación de la política de formación de profesores en nuestro caso. Asimismo, con igual objetivo de evaluación de políticas públicas al interior de la institución, tenemos la tesis de doctorado de Darío Arredondo López, La tutoría a estudiantes de economía en la Universidad de Sonora (2008); desde la economía, mencionaremos el texto de Benjamín Burgos, Alejandro Mungaray y Juan Manuel Ocegueda (coordinadores), Estructura económica y demanda de educación superior en el noroeste de México (2003).

\section{Conclusión}

Se entiende que no pretendemos hacer un recuento exhaustivo, sino dar un panorama general de la bibliografía relacionada con nuestro tema de investigación para situar en dicho contexto nuestra posible aportación. Podemos observar que la publicación de investigaciones y trabajos sobre la universidad no solo se amplió, sino se diversificó. Al principio predominan textos históricos, de caso único, con hechuras de diversa calidad, a ellos se le suman otros desde perspectivas científicas diferentes a la historia, sobre problemas de la universidad o del conjunto del sistema de educación superior.
Se puede afirmar, entonces, que el fenómeno mundial de masificación y diversificación de la educación superior y que tuvo su expresión tardía en Sonora provocó, además de la profesionalización del trabajo académico, el incremento de estudios sobre la educación superior, no sólo de las universidades, sino también la diversificación de las temáticas. El fenómeno bibliográfico tal parece reproduce en pequeño un patrón internacional, donde según entendemos, hasta la aparición de la obra de Clark Burton, The higher education system (1983), lo que privaba eran estudios de caso, incluso nacionales, de corte sobre todo histórico. A partir de entonces, al comparar los sistemas de educación superior en los países miembros de la OCDE, no solo se incrementaron los estudios sino se amplió la temática y la aportación de las diversas ciencias sociales al estudio de la educación superior y sus componentes. Ese mismo papel catalizador lo vino a presentar en la bibliografía latinoamericana el trabajo Educación superior y Estado en Latinoamérica. Desafíos privados a predominio público (1991) de Daniel Levy.

\section{Referencias:}

\section{Libros:}

Aldaco, Lic. Guadalupe Beatriz 2002. "Universidad de Sonora (Nuestros Rectores)”. Hermosillo, Sonora, México: Universidad de Sonora.

Arredondo López Darío, 2008. "La tutoría a estudiantes de economía en la Universidad de Sonora", Edición electrónica gratuita. Texto completo en www.eumed.net/tesis/ 2008/jdal/

Ayala Encinas Claudio, 2000. “El fin de una ilusión (ensayo político-cultural sobre los años setentas en la Universidad de Sonora). Hermosillo, Sonora, México: Consejo Nacional para la Cultura y las Artes (CNCA).

Burgos Benjamín, Alejandro Mungaray y Juan Manuel Oceguera, 2003. Estructura económica y demanda de educación superior en el noroeste de México. México: ANUIES. 
Castellanos Moreno Miguel, 1981. Historia de la Universidad de Sonora (1938-1953). Hermosillo, Sonora, México: Editores, Arnulfo Castellanos Moreno, Gabriela Rosales Gutiérrez y la Secretaria de Prensa del STAUS. Hermosillo Sonora, Una segunda edición por Editorial de la Universidad de Sonora, Hermosillo, Sonora, México, 1991.

Castellanos Moreno Miguel, 1993. "Historia de la Universidad de Sonora (1953 -1967). Editores, Arnulfo Castellanos Moreno y Gabriela Rosales Moreno. Hermosillo, Sonora.

Castellanos Moreno Miguel, 2006. "Historia de la Universidad de Sonora (El STAUS y el desarrollo académico)” Tomo IV. Guadalajara, Jalisco: Edición personal.

Castellanos Moreno Miguel, 2007. Historia de la Universidad de Sonora (en una época de crisis) Tomo III. Guadalajara, Jalisco: Edición personal.

Castrejon Diez, Jaime. Pérez Lizaur Marisol, 1976. Historia de las Universidades Estatales. México: SEP, Edición Selecciones Tipográficas.

Cota Grijalva Sofía Dolores, 2015. Departamento de Lenguas Extranjeras: una visión de su desarrollo en la Universidad de Sonora. Hermosillo, Sonora, México: Universidad de Sonora, La Mirada del Búho, editorial.

De Santiago, José Antonio, 2011. Lodos de aquellos polvos. Guadalajara, Jalisco, México: Santa Paula Editorial.

Duarte Rodríguez Rubén, 2003. "Días de fuego (El movimiento universitario sonorense de los años 70". Hermosillo, Sonora, México: Universidad de Sonora y Germinal.

Durand Villalobos Juan Pablo, 2006. "Poder, gobernabilidad y cambio institucional en la Universidad de Sonora (1991-2001). México D.F: ANUIES.

Estevez N. Etty Haydee, 1989. Panorámica de la investigación Educativa en la Unison (Primer Inventario de Proyectos). Hermosillo, Sonora, México: Editorial UNISONO.
Encinas Johnson Luis, 1954. Progreso y Problemas de México. México, Editorial Stylo.

Encinas Johnson Luis Lic., 1969. "la alternativa de México (conflictos, causas, caminos)”. México D.F.: Ediciones Sonot.

Esquivel Casas, Prof. Aureliano, 1942. "Posición Pedagógica Social y Filosófica de la Universidad. Hermosillo, Sonora, México: Edit. Universidad de Sonora.

Galaz A. Fernando, 1971. "Dejaron Huella en el Hermosillo de ayer y de Hoy. Hermosillo, Sonora, México: Gobierno del Estado de Sonora, segunda edición, 1996.

García, Luis Enrique, 1992. Siete notas para las Bellas Artes. Hermosillo, Sonora, México: Editorial UniSon.

García, Luis Enrique, Amadeo Hernández C., Ernesto López Riesgo, Rodolfo Díaz Castañeda, Graciela Barraza Uriarte y Fernando Cota Madero, asesoría en fotografía de Guillermo Moreno, 1992. Memoria gráfica de la Universidad de Sonora, Hermosillo, Sonora, México: Universidad de Sonora.

García Luis Enrique, 1996. "Memoria gráfica del deporte universitario". Hermosillo, Sonora: Universidad de Sonora, Colección Memoria gráfica \#1, asesor fotográfico, Guillermo “memo” Moreno.

García Luis Enrique, 2006. “Memoria gráfica del teatro universitario (1954-2004). Hermosillo, Sonora, México: Universidad de Sonora.

Lagarda Lagarda Ignacio, 2007. El color de las Amapas: crónica de la guerrilla en la sierra de Sonora. Hermosillo, Sonora, México, Liceo Tecnológico de Sonora A.C., Instituto Tecnológico Superior de Cajeme, Universidad tecnológica del Sur de Sonora.

Levy C., Daniel, 1991. Educación superior y el Estado en Latinoamérica. Desafíos privados a predomino público. México, Colección problemas educativos de México. FLACSO_-UNAM, edición en español. 
Macías Enríquez Alberto, 2006. "Historia de la facultad de derecho de la Universidad de Sonora”. Editorial Garabatos SA de CV. Hermosillo, Sonora, (segunda edición).

Mendivil Rincón, José Abraham, 1975. La Universidad de Sonora: Desde_su fundación. Hermosillo, Sonora: Publicidad Mendivil.

Mercado Andrews, Ismael, 1973. “El Día que Exploto la rabia (Huelga 1967 en Sonora). s.e. Folleto de Hermosillo Sonora.

Moncada Ochoa Carlos, 1988. La sucesión política en Sonora 1817-1985.México: Editorial latinoamericana.

Moncada Ochoa, Carlos, 1997. Diez en el poder. México: Edamex.

Moncada Ochoa Carlos, 2005. Historia General de la Universidad de Sonora, Tomo I, El principio del principio 1938-1953. Hermosillo, Sonora, México: Editorial Uni-Son.

Moncada Ochoa Carlos, 2006. Historia General de la Universidad de Sonora, Tomo II, legislación universitaria 1938-1991. De la autonomía formal a la autonomía real. Hermosillo, Sonora, México, Editorial Uni-Son.

Moncada Ochoa, Carlos, 2007. Historia General de la Universidad de Sonora, Tomo III, crecimiento, política y crisis 1953-1973. Hermosillo, Sonora, México: Editorial Uni-Son.

Moncada Ochoa Carlos, 2009. Historia General de la Universidad de Sonora, Tomo IV, la encrucijada 1973-1992. Hermosillo, Sonora, México: Editorial Uni-Son.

Moncada Ochoa Carlos, 2009. Historia General de la Universidad de Sonora, Tomo V, hacia la excelencia 1992-2009. Hermosillo, Sonora, México: Editorial Uni-Son.

Montoya Lara Joel, 1996. “Universidad Espacio y Arquitectura (Memoria Gráfica de la Arquitectura de la Universidad de Sonora). Hermosillo, Sonora, México: Universidad de Sonora.
Moreno Soto Armando, Ochoa Valenzuela Fernando, Miranda Miguel Darío y Bracamontes Sierra Álvaro, 1985. "Los Aguiluchos (Movimiento Popular Estudiantil de 1967 en Sonora). Hermosillo, Sonora, México: Universidad de Sonora.

Moreno Soto, Armando, 1991. "Historia del desarrollo de la Universidad de Sonora. Hermosillo, Sonora, México: edit. UNISONO, Colección No. 12.

Quiroz Martínez Manuel Prof., 1949. Monografía Histórica de la Universidad de Sonora”. Hermosillo, Sonora, México. Ponencia presentada en la "Novena sesión plenaria del Congreso Mexicano de Historia”. Hermosillo, Sonora. Diciembre 1949 (versión mecanográfica). Hermosillo, Sonora, México: Universidad de Sonora, primera edición 1999.

Rodríguez Mijangos Ricardo, 2015. Memorias alrededor de la investigación científica en Física en la Unison. Hermosillo, Sonora, México: Universidad de Sonora, La Mirada del Búho, editorial.

Sortillón Valenzuela Manuel de Jesús Ing., 1998. "Memorias de la Universidad de Sonora y de su escuela de Ingeniería”. Hermosillo, Sonora, México: Universidad de Sonora.

Uribe García Jesús Félix, 2001. Universidad de Sonora. El Museo, Hermosillo, Sonora México: editorial UniSon.

Vasconcelos José, 1998. El desastre. México: Editorial Trillas (primera edición).

Velarde Romero José Prof., s/f. "El movimiento magisterial en Sonora (El ardiente verano de 1967)". Hermosillo, Sonora, México: Ediciones la Voz.

Verdugo Córdova Joel, 2004. “El movimiento estudiantil en la Universidad de Sonora de 1970 a 1974 (un enfoque socio histórico a partir del testimonio oral). El Colegio de Sonora, Hermosillo, Sonora, México.

Vidal Alonso, 1999. Los nuestros (a propósito de centenarios). Hermosillo, Sonora, México, editora la voz de Sonora S.A. de C.V. 


\section{Capítulo de libro:}

Arredondo López, José Darío 2007. Economía y educación. El tiempo de la industrialización dependiente y el nuevo espacio universitario. En Darío Arredondo (coordinador), Tiempo y Espacio. Hermosillo, Sonora, México: Editorial Universidad de Sonora, 107-113.

\section{Artículos de revista:}

Casillas; Miguel. Notas sobre el proceso de transición de la universidad tradicional a la moderna. Los casos de la expansión institucional y la masificación. Sociológica Núm. 5, UAM, Azcapozalco, otoño de 1987, México, pp. 121-144.

Castellanos Moreno Miguel, abril de 1992. "Nacimiento del Departamento de Ciencias Sociales”. Revista Versiones, Hermosillo, pp. 11-13.

Clark R. Burton 1994. Diversificación de la educación superior: viabilidad y cambio. Universidad futura, vol. 5 \# 14, Universidad Autónoma MetropolitanaAzcapotzalco. México.

Molina, Freaner, Gustavo. Carrión Contreras, Enrique. Larios Velarde, Rodolfo. León Enríquez, Celina. Hernández López, Saúl, diciembre de 1985. “Modelo de Departamentalización de la Universidad de Sonora”. México DF: Comité de Educación Pública \#3, ANUIES, pp.109-124.

Moncada Ochoa, Carlos. Vida política de Herminio Ahumada. Lecturas de la lechuza, viernes 30 de enero de 1998. Hermosillo, Sonora.

Moreno Soto, Armando abril de 1988. Universidad de Sonora: cuarenta y cinco aniversarios de vida. Revista de la Universidad de Sonora \# 6, pp. 10-22. Reproducido bajo el título, larga transición de la universidad. Hermosillo Sonora, México: UniSono \# 65, 31 de octubre 1 de 1990.

Moreno Soto, Armando 1998 "Herminio Ahumada Ortiz y la fundación de la Universidad de Sonora”. Revista Universidad nueva época \#12, Hermosillo, Sonora, Universidad de Sonora, diciembre de 1998. Pp. 44-46.
Rico, María Guadalupe. “Higinio Blatt, Sociedades de la academia de política de la Universidad de Sonora, Revista de la Universidad de Sonora, Nueva época \#2, noviembre de 1994. Pp. 39-40.

Rodríguez, Raúl 1991. “Cronología de la reforma a la ley orgánica de la Universidad de Sonora”. Foro universitario 95, diciembre de 1991.

Rodríguez; Raúl (1997). “Origen y desarrollo de la educación superior en Sonora”. Estudios Sociales, vol. VIII, núm. 13, enero-junio de 1997. Hermosillo, Sonora, México; CIAD, ColSon, UniSon.

\section{Ponencias:}

Cortez Román, Nolvia 1997. Del desierto al Zócalo...Crónica del movimiento estudiantil de la Universidad de Sonora, 1991-1992. Hermosillo, Sonora: Memoria del XXII Simposio de Historia y Antropología de Sonora.

Díaz Castañeda, Rodolfo (1987). “Esbozo histórico de la Universidad de Sonora". Hermosillo, Sonora, México: Memoria XII Simposio de Historia y Antropología.

Encinas Blanco, Ángel 1998. Vida deportiva del lic. Herminio Ahumada Ortiz. Hermosillo, Sonora: Lecturas de la lechuza, viernes 30 de enero de 1998.

Encinas Johnson, Lic. Luis 20 de abril de 1958. " $\mathrm{La}$ Universidad de Sonora, Centro cultural del Noroeste”. Hermosillo Sonora: ponencia formulada por la Universidad de Sonora, entregada al Presidente de la República, Lic. Adolfo López Mateos.

Guillén Lugigo Manuela, 1997. Los no protagonistas: un testimonio del movimiento estudiantil de 1973. Hermosillo, Sonora: Memoria del XXII Simposio de Historia y Antropología de Sonora.

Jardines Moreno José Luis, 1997. ¿Rabia incontenida o candor desbordado? Hermosillo, Sonora: Memoria del XXII Simposio de Historia y Antropología de Sonora.

Larios Gaxiola Jesus., 1997. El movimiento estudiantil del 67 ¿Mito o verdadero movimiento social? Hermosillo, Sonora: Memoria del XXII Simposio de Historia y Antropología de Sonora. 
Mendez Sainz, Eloy. "Proyecto de universidad y de ciudad en Hermosillo de los cuarenta”. Memoria XVII Simposio de Historia y Antropología de Sonora. Universidad de Sonora, Hermosillo, febrero de 1992.

Ontiveros Almada Jorge, 1997. Crónica de un domingo. Hermosillo, Sonora: Memoria del XXII Simposio de Historia y Antropología de Sonora.

\section{Trabajos mimeografiados:}

Comité Pro-Casa de Estudiantes (CP-CE), junio 1985. Comisión de Orientación ideológica y política. "Situación Actual del Movimiento Estudiantil en la UNISON”. Hermosillo Sonora: mimeografiado.

Duarte Rodríguez, Rubén 1985. “Universidad y Luchas de Clases”. Hermosillo, Sonora: Versión Mimeografiada.

Encinas Johnson, Lic. Luis, Salazar Girón, Prof. Ernesto, Lic. José María Oceguera, Prof. Ernesto López Riesgo, Dueñas D, Ing. Alejandro, Hernández Muñoz, Ramón, González V, Fernando, Ahumada B, Arnoldo. La Universidad de Sonora, Centro Cultural del Noroeste, s.e. (versión mecanográfica).

Ferrá Martínez, Carlos. El movimiento Universitario Sonorense de 1973. s.e. Hermosillo Sonora: (versión mecanográfica).

Larios Velarde Luis Felipe. Gurrola Mac Enrique y Salido Ontiveros Jesús Alejandro 1985. “Notas Históricas sobre la creación de la Universidad de Sonora en 1938”. s.e. México, D.F. diciembre de 1985.

Moreno Soto, Armando. "El Movimiento Estudiantil Sonorense de 1970-1973. Inédito Hermosillo, Sonora. (Versión mecanográfica).

Ramírez, José Luis 1994. The Change of the University of Sonora Law: A Case Analysis, s.e Tucson, Arizona, julio 13 de 1994 (versión mecanográfica).

Reyes, Ana Teresa. “Cronología de la reforma a la ley orgánica de la Universidad de Sonora”. Hermosillo, Sonora.

\section{Periódicos:}

Gutiérrez Mendivil, Domingo. 13 de octubre de 1982 al 18 de marzo de 1983. "La Universidad de Sonora en sus Orígenes, Hermosillo, Sonora México: periódico Información.

Moreno Soto, Armando. El Movimiento Estudiantil y Popular de 1967 en Sonora. Periódico Información. Hermosillo, Sonora, 26, 27, 28, 29 de septiembre de 1983. Hermosillo, Sonora. 